\title{
Consideraciones en torno a los tumores del mediastino
}

\author{
Considerations about mediastinal tumours
}

\author{
Ibrahima Kalil Keita ${ }^{1} \mathbb{D}$, Ana María Nazario-Dolz² $\mathbb{D}$, Gilberto Carlos Falcón-Vilariño $\mathbb{D}^{\mathbb{D}}$, \\ Luis Castillo-Toledo ${ }^{4} \mathbb{D}$, Zenén Rodríguez-Fernández ${ }^{5} \mathbb{D}$, Lázaro Ibrahim Romero-García ${ }^{6}$
}

1 MD, especialista Cirugía General y en Medicina General Integral, Hospital Provincial Docente "Saturnino Lora", Santiago de Cuba, Cuba.

2 MD, especialista en Cirugía General, magister en Urgencias médicas, Hospital Provincial Docente "Saturnino Lora"; profesora e investigadora auxiliar, Universidad de Ciencias Médicas, Santiago de Cuba, Cuba.

3 MD, especialista en Cirugía General, magister en urgencias médicas, Hospital Provincial Docente "Saturnino Lora"; profesor asistente, Universidad de Ciencias Médicas, Santiago de Cuba, Cuba.

4 MD, especialista en Otorrinolaringología, magister en urgencias médicas, profesor auxiliar, Hospital Provincial Docente "Manuel Ascunce Domenech", Camagüey, Cuba.

$5 \mathrm{MD}$, especialista en Cirugía General, magister en Informática en Salud, profesor auxiliar y consultante, investigador auxiliar, Hospital Provincial Docente "Saturnino Lora" Santiago de cuba, Cuba.

6 MD, especialista en Bioestadística, magister en Salud Pública y en Epidemiología; investigador auxiliar, Hospital Provincial Docente "Saturnino Lora" Santiago de cuba, Cuba.

\section{Resumen}

Introducción. El desarrollo de las imágenes diagnósticas hizo posible el diagnóstico topográfico de los tumores de mediastino; sin embargo, aún con los avances actuales de la cirugía, la anestesiología y la reanimación, el acceso quirúrgico a ese espacio medio de la caja torácica continúa siendo el gran reto que enfrentan los cirujanos torácicos.

Objetivo. Profundizar en los diferentes aspectos cognoscitivos actualizados sobre los tumores mediastinales.

Métodos. Búsqueda documental digital en bases de datos: Scielo, Lilacs, Web of Science, PubMed, realizada en enero de 2019, de publicaciones sobre el tema en la presente centuria.

Resultados. Las neoplasias mediastinales son poco frecuentes, pueden aparecer a cualquier edad, aunque son más frecuentes entre la tercera y quinta décadas, y la mayoría se descubren incidentalmente en una radiografía de tórax de rutina en pacientes asintomáticos. Los tumores malignos del mediastino son poco frecuentes, pero los benignos constituyen un desafío diagnóstico para radiólogos y patólogos. En las neoplasias malignas se identifica una amplia gama de variedades histológicas, atribuibles a las características del órgano afectado.

Conclusiones. El diagnóstico definitivo generalmente se establece mediante el estudio histopatológico posquirúrgico, si bien la tomografía computarizada, asociada o no a la biopsia percutánea, es el estándar de oro para el diagnóstico preoperatorio. La selección de la vía de entrada al tórax, así como el procedimiento quirúrgico, están condicionados por la localización y el tamaño del tumor en el mediastino.

Palabras clave: mediastino; neoplasias del mediastino; diagnóstico; cirugía torácica; esternotomía; endoscopía.

Fecha de recibido: 08/09/2019 - Fecha de aceptación: 28/01/2020

Correspondencia: Ibrahima Kalil Keita, Calle Reloj 158 entre Habana y Trinidad, Santiago de Cuba, Cuba.

Teléfonos: +5322655222 Celular: +5353737112

Correo electrónico: lilkeith8681@yahoo.com

Citar como: Keita IK, Nazario-Dolz AM, Falcón-Vilariño GC, Castillo-Toledo L, Rodríguez-Fernández Z, Romero-García LI. Consideraciones en torno a los tumores del mediastino. Rev Colomb Cir. 2020;35:472-82. https://doi.org/10.30944/20117582.460

Este es un artículo de acceso abierto bajo una Licencia Creative Commons - BY-NC-ND https://creativecommons.org/licenses/by-ncnd/4.0/deed.es 


\begin{abstract}
Introduction. The development of diagnostic images made the topographic diagnosis of mediastinal tumors possible; however, even with current advances in surgery, anesthesiology, and resuscitation, surgical access to this mid-rib cage space continues to be the great challenge faced by chest surgeons.
\end{abstract}

Objective. To deepen in the different updated cognitive aspects about mediastinal tumors.

Methods. Digital documentary search in databases: Scielo, Lilacs, Web of Science, and PubMed, performed in January 2019, of publications on the subject in the present century.

Results. Mediastinal neoplasms are rare, they can appear at any age, although they are most common between the third and fifth decades, and most are discovered incidentally on a routine chest radiograph in asymptomatic patients. Malignant tumors of the mediastinum are rare, but benign tumors pose a diagnostic challenge for radiologists and pathologists. In malignant neoplasms, a wide range of histological varieties is identified, attributable to the characteristics of the affected organ.

Conclusions. The definitive diagnosis is generally established by postoperative histopathological study, although computed tomography, associated or not with percutaneous biopsy, is the gold standard for preoperative diagnosis. The selection of the entry route to the thorax, as well as the surgical procedure, are conditioned by the location and size of the tumor in the mediastinum.

Key words: mediastinum; mediastinal neoplasms; diagnosis; thoracic surgery; sternotomy; endoscopy.

\section{Introducción}

El mediastino se desarrolla del endodermo, mesodermo y ectodermo, a partir de la cuarta semana de gestación, y en la séptima semana se fusiona la membrana pleuropericárdica con el mesodermo ventral del esófago, cuya posterior evolución determina el completo desarrollo mediastínico, que es el compartimiento central, extrapleural del tórax, que separa las dos cavidades pleurales, ubicado por detrás del esternón, por delante de la columna vertebral y por encima del diafragma.

El mediastino alberga múltiples estructuras anatómicas. Tiene características embriológicas, fisiológicas y anatómicas particulares, pues a través de él pasan sangre, linfa, aire y alimento ingerido; es, además, el área donde mayor actividad tienen los nervios autónomos. A este nivel ocurre el desarrollo embriológico de los sistemas circulatorio, respiratorio y digestivo, por lo que pueden presentarse malformaciones congénitas de diversa índole, que, junto a procesos inflamatorios, traumáticos y neoplásicos, hacen de este un compartimiento muy complejo, donde se presenta una amplia variedad de enfermedades ${ }^{1-5}$.
El crecimiento de tumores en el mediastino causa compresión de las estructuras vecinas, como la médula espinal, vasos sanguíneos y esófago, además de la diseminación a estructuras circundantes, como el corazón y los grandes vasos. Sus complicaciones también se derivan del tratamiento, sea por cirugía, radioterapia o quimioterapia ${ }^{5-7}$.

Dada su situación anatómica, el mediastino permaneció durante siglos como un territorio inexplorado, pero con el advenimiento de la radiología, se comenzaron a diagnosticar los tumores de mediastino; sin embargo, por mucho tiempo existió el valladar de su inaccesibilidad operatoria. El acceso quirúrgico a ese espacio medio de la caja torácica ha sido siempre el gran problema al que se han enfrentado los cirujanos torácicos ${ }^{7-9}$.

Teniendo en cuenta esas premisas y el aumento del número de enfermos operados con tumores mediastinales malignos en nuestro entorno ${ }^{10-12}$, se decidió realizar una revisión bibliográfica, con el objetivo de profundizar en sus diferentes aspectos cognoscitivos actualizados sobre este importante tema. 


\section{Métodos}

Mediante la técnica de búsqueda documental digital en diferentes bases de datos: Scielo, Lilacs, Web of Science, PubMed, efectuada en enero de 2019, se localizaron publicaciones de la presente centuria sobre el referido tema, utilizando las siguientes palabras clave: mediastino, compartimientos mediastinales, tumores mediastinales, diagnóstico, tratamiento, esternotomía.

\section{Revisión del tema}

\section{Algunos aspectos históricos}

Desde I787, en el diccionario de ciencias y artes se registra una definición del "espacio mediastinal" y otra curiosa acepción, donde lo detallan como una doblez de la pleura, que divide el pecho en dos mitades y viene desde la espalda ${ }^{\mathrm{I}, \mathrm{r} 3}$.

Pocos cirujanos, a finales de I80o y principios de 1900 , intentaron describir enfoques quirúrgicos para el mediastino ${ }^{5,13}$. En I888, Nassiloff demostró que al esófago podía llegarse mediante un acceso posterior, tomando como referencia el tratamiento empleado en pacientes con perforación. Años después, otros cirujanos comenzaron a acercarse a anomalías del mediastino anterior. En I893, Bastinelli describió la extracción de un quiste dermoide mediastínico anterior y realizó una resección del manubrio esternal. En I896, Ludwig Rehn de Frankfort utilizó el acceso quirúrgico transcervical ${ }^{1,14}$. Ya en I897, Milton escribió extensamente sobre la cirugía del mediastino al utilizar las ventajas de la esternotomía media, y encontró que le daba un excelente acceso a esa localización. Las mediastinotomías anteriores fueron descritas en I90I por ese autor en Inglaterra y por Ricard en Francia ${ }^{5,14}$.

La primera operación de la miastenia grave fue realizada por Sauerbruch el 6 de marzo de I9II. A través de un acceso transcervical operó a una mujer de I9 años de edad que padecía esa afección y bocio coexistente. Extrajo un tumor de $49 \mathrm{~g}$ de timo hiperplásico y observó mejoría posterior del cuadro clínico. En los siguientes 20 años hubo informes intermitentes de cirujanos que ejecutaron esta intervención, con buena respuesta en términos de mejoría sintomática ${ }^{\mathrm{I}, \mathrm{I}}$.

La era moderna de la cirugía tímica comenzó con Alfred Blalock en el John's Hopkins Hospital en 1936, quien a través de una vía transesternal extirpó un tumor del timo en un paciente con miastenia grave y sugirió que la exploración de la región tímica estaría indicada en todos los pacientes con miastenia. Unos años más tarde, en I94I, aplicó esta teoría e introdujo la timectomía en casos de pacientes miasténicos sin timoma, con mejoras similares. En 1944 comunicó su serie de 20 pacientes y así comenzó a aplicarse una nueva opción terapéutica para combatir la miastenia; pero ya en 1940 Heuer, quien había publicado una monografía sobre los tumores del mediastino, también describió la eliminación exitosa de tumores neurogénicos del mediastino posterior y las características de varios tipos de tumores del timo ${ }^{15,16}$.

En 1959, Carlens realizó una mediastinoscopia transcervical, utilizando el mediastinoscopio creado por él. Mucho más tarde Joel Cooper, en 1988, desarrolló el retractor del manubrio esternal, que permite una mejor visualización del mediastino anterior y exposición de la glándula, lo que hace posible una completa resección del timo desde el cuello; modificación que de nuevo hizo popular el acceso transcervical. La utilización de un videoscopio contribuye a mejorar la visión y disección anatómica ${ }^{5,17-19}$.

\section{Detalles anatómicos el mediastino}

Existen varias clasificaciones que dividen el mediastino en diferentes compartimientos, basadas en la anatomía topográfica o quirúrgica y en la radiología. Según Shields y Felson, se distingue un compartimiento anterior, uno medio y otro posterior. El Dr. José L. Martínez propuso la división del mediastino en 9 compartimientos: previsceral: superior medio e inferior; visceral: superior, medio e inferior, y retrovisceral: superior, medio e inferior ${ }^{1,5}$.

Actualmente, la más usada es la clasificación que lo divide en mediastino superior e inferior y este a su vez en anterior, medio y posterior ${ }^{9,14}$. 
I. Mediastino superior: Se extiende desde la apertura torácica superior hasta el plano horizontal que pasa por el ángulo esternal y el borde inferior de la IV vértebra torácica. Contiene, de delante hacia atrás, las siguientes estructuras: origen de los músculos esternohioideo y esternotiroideo, timo y sus restos, venas braquiocefálicas, mitad superior de la vena cava superior, arco aórtico, tronco arterial braquiocefálico, arteria carótida común izquierda, arteria subclavia izquierda, nervios frénicos, nervios vagos, nervio laríngeo inferior izquierdo o recurrente, tráquea, esófago, conducto torácico, terminación de los músculos largos del cuello, tejido areolar y graso.

2. Mediastino inferior: Se sitúa por debajo del superior. Este a su vez se subdivide por la presencia del corazón en anterior, medio y posterior.

a) Mediastino anterior: Situado delante del pericardio, detrás del esternón y por encima del diafragma. Contiene vasos mamarios internos, extremo inferior del timo, tejido areolar graso y ganglios linfáticos.

b) Mediastino medio: Es la región más amplia del mediastino inferior y contiene el corazón y su pericardio, la aorta ascendente, arterias y venas pulmonares, bronquios principales, cava superior, nervios frénicos y otras estructuras.

c) Mediastino posterior o espacio retrocardíaco de Helzknecht. Está limitado al frente por el corazón, detrás por las últimas ocho vértebras dorsales, encima por el mediastino superior y debajo por el diafragma. Contiene el esófago, la aorta descendente torácica, los nervios neumogástricos, simpáticos e intercostales, el conducto torácico, las venas ácigos y hemiácigos, las venas ácigos accesoria, cadenas simpáticas, ganglios linfáticos, tejido areolar y graso.

El mediastino recibe el drenaje linfático del cuello, la pared torácica anterior, los pulmones, el esófago, el pericardio, el diafragma y la cavidad abdominal ${ }^{1,5,13}$. Los ganglios mediastinales se dividen en:

Grupo anterior: Tiene tres cadenas ganglionares, todas en el mediastino superior, dos verticales y una transversa, que se anastomosa a las dos primeras.

Grupo medio: Ganglios intertraqueobronquiales (situados debajo de la bifurcación traqueal), los peritraqueales y los del pedículo pulmonar.

Grupo posterior: Ganglios aorticoesofágicos (situados a lo largo del esófago).

La localización de un tumor en uno u otro compartimento orientará hacia la naturaleza de dicha lesión, unido a la clínica del paciente y las características imagenológicas ${ }^{5,10}$.

\section{Aspectos fisiológicos del mediastino}

Las funciones del mediastino son múltiples, entre las cuales figura ser amortiguador de doble membrana, al estar sometido a la influencia de la presión negativa que se encuentra en ambas cavidades pleurales, lo que le permite, en condiciones patológicas, pendular hacia el lado de menor presión. El mediastino responde a estos hechos manteniendo una función normal de los órganos. También tiene función hemodinámica, pues al ser negativa en $-3 \mathrm{~mm}$ $\mathrm{Hg}$, la presión mediastínica permite la aspiración torácica.

Las funciones accesorias son diversas: acción sobre el ritmo respiratorio y cardiaco, acción sobre la función respiratoria, acción sobre la presión arterial, acción sobre la motilidad cardiaca y el débito coronario, acción sobre el sistema neurosensitivo, función de fagocitosis y de fijación tisular, relaciones mediastino-endocrinas y mediastino-neurotróficas ${ }^{\mathrm{I}, \mathrm{14}}$. 


\section{Algunos aspectos clínico- epidemiológicos}

Aproximadamente $40 \%$ de los pacientes con tumores en el mediastino son asintomáticos. Los timomas y los carcinomas tímicos constituyen las neoplasias más comunes del mediastino ${ }^{3,10, \mathrm{II}}$. Los timomas representan el $50 \%$ de los tumores del mediastino anterior y entre $15-20 \%$ del total de los tumores mediastinales. En los niños ${ }^{20,21}$, los tumores neurogénicos del mediastino posterior son los más frecuentes y representan casi $50 \%$ de los tumores mediastinales, en contraste con los adultos, en los cuales el timoma en el mediastino anterior resulta el más común ${ }^{\mathrm{I}, \mathrm{II}}$.

Los linfomas representan $20 \%$ de todas las neoplasias mediastinales en los adultos y $50 \%$ en los niños ${ }^{20,21}$. Los tumores de células germinales del mediastino constituyen IO-I5 \% de los tumores mediastinales, y esos mismos porcentajes de las tumores mediastinales anteriores ${ }^{22-27}$. Los quistes mediastinales causan 20-32 \% de los tumores mediastinales primarios, y entre ellos, los quistes broncogénicos representan 50-60 \% ${ }^{28,29}$. Los tumores mesenquimáticos se diagnostican en Io \% de los tumores mediastínicos, y son más frecuentes en la población pediátrica; entre estos, los lipomas resultan los más frecuentes ${ }^{30-32}$. La enfermedad de Castleman puede afectar cualquier grupo o territorio ganglionar, pero mayormente se origina en el tórax $(70 \%)^{33}$.

No son infrecuentes los linfomas, las afecciones tumorales de las glándulas tiroides y paratiroides, los teratodermoides, el carcinoma primario del mediastino, los tumores de células germinales, los hamartomas, plasmocitomas y tumores de origen linfático, principalmente en la parte anterior (representan alrededor de I7\%). Allí los más comunes son los linfomas de Hodgkin, variedad esclerosis nodular, que son de mejor pronóstico; mientras los linfomas no Hodgkin, con una presentación clínica similar, son de evolución agresiva y mala pronóstico ${ }^{33-36}$.

Las afectaciones localizadas incluyen quistes y tumores primarios, infecciones, hemorragias, enfisema y aneurismas; las enfermedades sistémicas: los tumores metastásicos y los trastornos granulomatosos e inflamatorios. Las lesiones originadas en esófago, en grandes vasos, tráquea y corazón se manifiestan como una masa y establecen un diagnóstico diferencial con la enfermedad primaria del mediastino ${ }^{2, \mathrm{II}-\mathrm{I} 4,37}$.

\section{Manifestaciones clínicas}

Las afecciones quirúrgicas del mediastino devienen un tema controversial, por la variedad de las manifestaciones clínicas, compromiso de estructuras adyacentes y complejidad de las intervenciones quirúrgicas a realizar. ${ }^{\mathrm{I}, 2,8,} \mathrm{~A}$ pesar de la afectación local, la intervención de factores secretores tumorales e inmunológicos y las múltiples enfermedades sistémicas que pueden acompañarlo, ocasionalmente se presentan de forma asintomática, solo como hallazgo incidental en estudios radiográficos ${ }^{6,10,37}$.

El cuadro sintomático es muy florido; sin embargo, existe un porcentaje no despreciable de pacientes asintomáticos, de manera tal que la identificación de la presencia del tumor en estos pacientes suele ser fortuita, casi siempre a través de una radiografía de tórax.

Las manifestaciones dolorosas son las más comunes. En general el dolor es retroesternal, con irradiación al dorso, o bien erosivos óseos, o de tipo neurálgico o braquiálgico, propios de los tumores nerviosos, en particular con prolongación intramedular. También puede originarse por extensión tumoral a la pared torácica, pleura, diafragma $u$ otros sitios, lo que sería indicativo de malignidad ${ }^{\mathrm{1}, 2, \mathrm{II}}$.

De igual forma, hay manifestaciones circulatorias, que suelen ser las más aparentes, y se generan por compresión de la circulación venosa de retorno, lo que ocasiona ${ }^{32,34,35,37}$ :

$\checkmark$ Edema de cuello y cara, sobre todo en los párpados.

$\checkmark$ Inflamación y turgencia de las venas, inicialmente en la base de la lengua, que aumenta al hablar, toser, deglutir y al inclinar la cabeza y el tórax hacia delante.

$\checkmark$ Cianosis y circulación colateral son los signos más tardíos. Afectan la región anteroto- 
rácica, el hombro y la base del cuello; pero si compromete la vena ácigos, aparece circulación toracoabdominal muy manifiesta. Este síndrome se conoce con el nombre de síndrome de la vena cava superior. En general es producido por enfermedades malignas y, por tanto, su pronóstico es malo; sin embargo, algunos pocos casos pueden tener un origen benigno, ya sea por enfermedades vasculares (fístula arteriovenosa, aneurisma aórtico, trombosis de la vena cava superior) u otras (tuberculosis, hematoma traumático, fibroma quístico, tiroides retroesternal).

El cuadro clínico respiratorio por lo común consiste en tos seca y disnea con predominio inspiratorio ${ }^{12,13,28}$. Los síntomas digestivos suelen ser atribuibles a la compresión del esófago; el síntoma fundamental es la disfagia, casi siempre tardía, intermitente y a veces paradójica (mayor para líquidos que para sólidos) ${ }^{14,1734}$.

Además, puede haber manifestaciones neurológicas, capaces de presentarse en forma de neuralgia aguda o bien de parálisis motoras. Así, la afección del neumogástrico causa sialorrea, bradicardia, disnea y tos. La parálisis del simpático se manifiesta por el síndrome de Claude Bernard-Horner (ptosis palpebral, miosis, enoftalmia y estrechamiento de la abertura palpebral). En los tumores neurógenos puede producirse la afección medular por compresión, pero es rara. Por último, en el síndrome de Tobias-Pancoast son frecuentes las braquialgias por invasión del plexo braquial ${ }^{17,32,36,37}$.

Las manifestaciones parietales son raras y se observan sobre todo en la infancia, como abombamiento con deformación torácica o invasión neoplásica de la pared. En los quistes y tumores benignos son menos frecuentes, porque la compresión permite el desplazamiento de los elementos mediastinales; por el contrario, resultan más comunes en los malignos, porque se añade la fijación e invasión del tumor a las estructuras ${ }^{\mathrm{I}, 16}$.

\section{Diagnóstico}

La ubicación más común de los tumores mediastinales depende de la edad del paciente. ${ }^{9,35,36}$ Por ejemplo, en los niños son más comunes en el compartimiento posterior y suelen ser de origen neurogénico y benignos, ${ }^{20,21,25,32}$ mientras que, en los adultos (más comúnmente de la tercera a la quinta décadas de la vida) se forman en el compartimiento anterior, y constituyen habitualmente linfomas o timomas malignos ${ }^{37}$.

En la práctica diaria es posible diagnosticar estas entidades mediante el estudio anatomopatológico, pero resulta primordial la combinación de hallazgos clínicos e imagenológicos junto con exámenes auxiliares como la inmunohistoquímica, la microscopia electrónica y modernamente, el análisis cromosómico ${ }^{24}$. El desarrollo tecnológico ha permitido que el diagnóstico sea más frecuente y certero ${ }^{6,14,18,19}$.

Ante un paciente con un tumor mediastinal debe establecerse un diagnóstico diferencial entre las diversas causas que lo originan. Para ello se impone tener en cuenta los siguientes aspectos:

I. La edad: en los niños, los tumores neurogénicos y los quistes enterogénicos son las masas mediastínicas más frecuentes; en los adultos, los tumores neurogénicos, linfomas y quistes tímicos. ${ }^{2}$

2. Los síntomas;

3. Las pruebas de laboratorio: se solicitan ante la sospecha de determinados tumores, por ejemplo: pruebas de función tiroidea, en pacientes con bocio endotorácico; niveles de hormonas paratiroideas; anticuerpos antireceptor de acetilcolina, para descartar miastenia grave en pacientes con sospecha de timoma, aunque estén asintomáticos; $\alpha$-fetoproteinas y $\beta$-gonadotropina coriónica humana, excreción de ácido vanilmandélico y catecolaminas en orina de 24 horas, en el feocromocitoma, paraganglioma y neuroblastoma; entre otros ${ }^{2,8,10,15}$.

4. Y por supuesto, las imágenes y procedimientos diagnósticos, a saber ${ }^{6,7}$ :

- Radiografía simple de tórax. Es la prueba diagnóstica inicial, que muestra dónde está la lesión. Las proyecciones poste- 
roanterior y lateral, y a veces las oblicuas, permiten evaluar el tamaño, la localización y la densidad del tumor, así como la presencia de calcificaciones en la masa.

- Tomografía computarizada (TC) de tó$\operatorname{rax}^{1,37}$. Es un estudio de rutina en los pacientes con una masa mediastinal, que posibilita determinar: situación, tamaño, forma, densidad y composición del tumor, presencia y tipo de calcificaciones, características del borde del tumor, relación con las estructures vecinas, presencia y localización de linfadenopatías, y otros hallazgos asociados; además de que es capaz de diferenciar las masas sólidas de las quísticas. También permite discriminar el contenido de las zonas ocultas del mediastino en las radiografías convencionales, debido a la superposición de las opacidades esternal, cardíaca y de la columna vertebral.

- Resonancia magnética nuclear ${ }^{1,5,54}$. Está indicada en el estudio de los tumores neurogénicos, para confirmar si existe o no afectación de estructuras nerviosas, en pacientes en los que no puede utilizarse el medio de contraste para la realización de la TC, por falla renal o alergia; para determinar si existe invasión vascular o del plexo braquial, $u$ otro proceso. Tiene la ventaja de no emitir radiaciones ionizantes, y puede ser más útil que la TC para evaluar la dilatación del foramen de conjunción en los tumores neurogénicos del mediastino posterior. También se ha mostrado superior para diagnosticar diferentes tipos de quistes.

- Ecografía. Diferencia lesiones quísticas y sólidas y sirve para dirigir la punción aspiración con aguja fina (PAAF). La ecografía transesofágica se emplea en el estudio de lesiones esofágicas y estructuras paraesofágicas ${ }^{6,7, \mathrm{IO}}$. Puede ser útil para evaluar tumores situados en el mediastino medio, y en pacientes con taponamiento cardíaco o estenosis pulmonar.
- Isótopos ${ }^{\mathrm{I}, \mathrm{I4}}$. Tiene una sensibilidad de 93 $\%$ y una especificidad de $100 \%$ en el diagnóstico del bocio endotorácico si existe tejido tiroideo funcionante, para detectar linfomas y diferenciarlo de los timomas, o para localizar mucosa gástrica ectópica, entre otras ventajas.

- Tomografía por emisión de positrones (PET) ${ }^{1,5,9,37}$. Es útil para el diagnóstico y estadificación del carcinoma broncogénico y los linfomas, y para seguimiento de tumores de células germinales tras el tratamiento.

Otras técnicas diagnósticas de importancia, sobre todo en caso de realización de biopsia por aspiración con aguja fina (BAAF) son:

- Broncoscopia ${ }^{28,29}$. Es válida para el estudio de lesiones del mediastino medio con afectación pulmonar asociada, y para realizar punción de adenopatías.

- Punción-aspiración con aguja fina. Tiene limitaciones, pues se necesitan muestras mayores de tejido. Está contraindicada cuando se sospecha un timoma, debido al elevado riesgo de diseminación por el trayecto de la aguja ${ }^{5,15}$.

- Biopsia. La técnica se realiza en función del lugar donde se localice la lesión. Si no es accesible por técnicas específicas, se impone ejecutar una toracoscopia, o mediastinoscospia.

En algunos casos, se puede realizar biopsias de médula ósea, de ganglios periféricos, de ganglios mediastinales, o de la masa, por aspiración transbronquial o transesofágica, con apoyo de ultrasonido y agujas especiales.

La biopsia por aspiración transtorácica con aguja fina, ayudada por un estudio de imagen, es un procedimiento sencillo, de gran sensibilidad para diagnosticar una neoplasia maligna, pero no para precisar el tipo de tumor. Por eso, frecuentemente se debe recurrir a mediastinostomía anterior, mediastinoscopia, toracoscopia o minitoracotomía, para tomar una muestra suficiente, de 
al menos I mL cúbico; en raras ocasiones se recurre a la mediastinostomía posterior. En caso de un tumor localizado, sin metástasis, con diagnóstico preoperatorio incontrovertible, es preferible proceder a la resección de la masa tumoral durante el mismo acto quirúrgico de la biopsia, ampliando el mismo acceso o realizando una esternotomía parcial o total ${ }^{5,10,16,17}$.

- Mediastinoscopia. Este estudio puede ser realizado con el equipo clásico o mediante el videomediastinoscopio, avance técnico que permite incorporar imágenes de video al instrumento convencional, a fin de obtener una mejor visión de las estructuras mediastinales. Igual sucede con el mediastinoscopio bivalvo, que amplia el espacio mediastínico y posibilita el trabajo bimanual, con la ayuda del asistente. En la actualidad, la videomediastinoscopia (VMC) o mediastinoscopia videoasistida es la modalidad preferida en la mayoría de las instituciones, por cuanto la variante clásica quedó relegada a un segundo plano ${ }^{\text {I8,19. }}$.

Otros estudios ${ }^{37}$ no son indispensables ni necesarios para el diagnóstico de los tumores mediastinales, pero complementan la información obtenida; por ejemplo, el esofagograma, la esofagoscopia, la broncoscopia, la ecobroncoscopia y el ecocardiograma, para evaluar el grado de contacto o invasión de estos órganos. Si se sospecha un tumor germinal, debe indicarse una TC abdominal para explorar el retroperitoneo, y una ecografía testicular, se palpe o no masa tumoral.

\section{Acceso quirúrgico del mediastino ${ }^{1,16,37}$}

Las principales vías de acceso quirúrgico al mediastino son:

I. Vía superior: vía de Carlens (incisión supraesternal), cervicotomia lateral izquierda

2. Vías torácicas:

a. Anteriores (intrapleurales): esternotomia media, esternotomia parcial (con incisión supraesternal o puerta de tram- pa). Esternotomia total o de Milton $\mathrm{Du}$ val y la vía de Chamberlain.

b. Toracotomías (intrapleurales): anterolateral (Rienhoff), posterolateral (Sweet), axilar vertical (Nuncio di Paola).

c Toracotomías posteriores (extrapleurales): vía paravertebral posterior derecha, ventana pleural.

3. Vía inferior: Retroesternal y preperitoneal, ventana pericárdica transdiafragmática, transhiatal.

\section{Tratamiento}

El tratamiento se sustenta sobre diferentes bases terapéuticas, pero para la gran mayoría, es de elección la cirugía, con excepción de los linfomas y los tumores de células germinales seminomatosos y algunos metastásicos, en cuyo caso la quimioterapia o radioterapia constituye la terapéutica de elección ${ }^{38-41}$. La escisión quirúrgica completa se considera lo ideal, aunque también está indicada la reducción en las lesiones de tamaño inicialmente inoperable.

En muchos pacientes con tumores de mediastino, el tratamiento quirúrgico es complejo y exige con frecuencia una técnica difícil (figura I) ${ }^{15,16,42}$. El conocimiento de la fisiología del mediastino y del control de la ventilación, así como de las nuevas técnicas anestésicas, permiten aplicar un tratamiento quirúrgico más seguro en esta región corporal, con lo cual se logra disminuir las tasas de morbilidad y mortalidad ${ }^{8,43}$.

\section{Consideraciones finales}

Las neoplasias mediastinales son poco frecuentes, pueden aparecer a cualquier edad, y la mayoría se descubren incidentalmente en una radiografía de tórax de rutina en pacientes asintomáticos. Los tumores malignos del mediastino son poco frecuentes, pero los benignos constituyen un desafío diagnóstico para radiólogos y patólogos. En las neoplasias malignas se identifica una amplia gama de variedades histológicas, atribuibles a las características del órgano afectado. 
A B
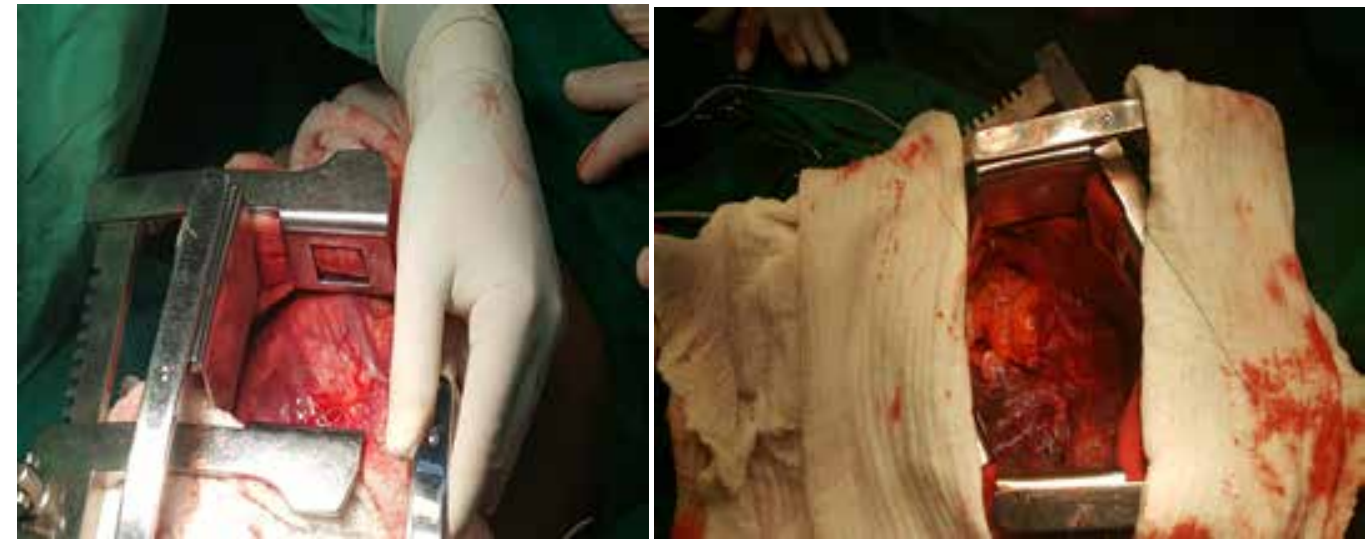

$\mathrm{C}$

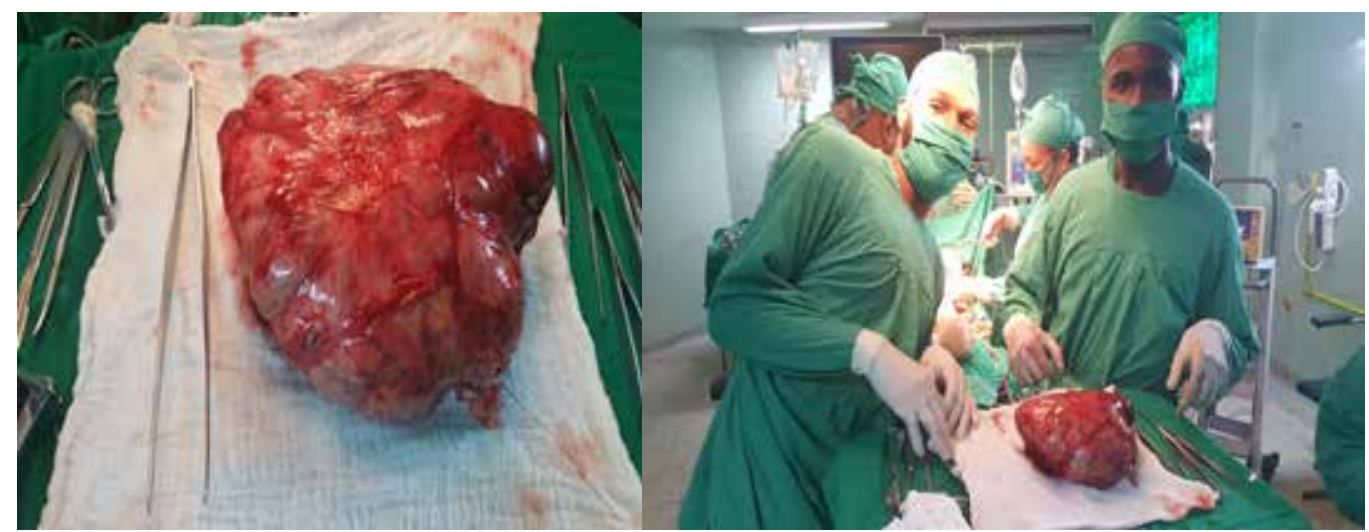

Figura 1. Imágenes de la extirpación de un tumor mediastinal gigante (timoma) en un paciente masculino de 45 años quien acudió a consulta por cansancio y disnea de esfuerzo. A: toracotomía anterolateral izquierda, se evidencia el tumor gigante en el mediastino medio. B: espacio anatómico que ocupaba el tumor, se evidencia el contacto directo con el miocardio y el pulmón izquierdo. C: Pieza quirúrgica obtenida, timoma de más de $10 \mathrm{~cm}$.

La tomografía computarizada, asociada o no a la biopsia percutánea, es el estándar de oro para el diagnóstico preoperatorio. El diagnóstico definitivo generalmente se establece mediante el estudio histopatológico posquirúrgico, con excepción de los linfomas y los tumores de células germinales seminomatosos y algunos metastáticos. La selección de la vía de entrada al tórax, así como el procedimiento quirúrgico, están condicionados por la localización y tamaño del tumor en el mediastino.

\section{Cumplimiento de normas éticas}

Consentimiento informado: Este estudio es una revisión de la literatura, y como tal no hay necesidad de un consentimiento informado ni de aprobación del Comité de Ética Institucional.

Conflicto de interés: Ninguno declarado por los autores.

Fuentes de financiación: Recursos propios de los autores. 


\section{Referencias}

I. Nason KS, Maddaus MA, Luketich JD. Pared torácica, pulmón, mediastino y pleura. En: Brunicardi FC. Schwartz Principios de cirugía. Io ed. México: McGraw-Hill Global Education; 2015. Fecha de consulta: I7 de diciembre de 20I8. Disponible en:

https://accessmedicina.mhmedical.com/content.aspx?bookid=I5I3\&sectionid

2. Torres-Rodríguez T, Herrera-Cruz D, Gálvez-González M, Moran-Ocaña E, del Cid-Herrera RM, Gordillo-Castillo R. Masas mediastinales: Epidemiología y decisiones estratégicas. Experiencia de 13 años. Neumol. Cir. Torax. 2016;75:268-74.

3. Ruiz-Cabrera YF, Ruiz-Cabrera Y, Rodríguez-Reyes SJ. Teratocarcinoma de mediastino. Medicentro Electrónica. 2015;19:118-2I.

4. Soomro NH, Pervaiz S, Hussain A, Ali SK. Solitary fibrous tumour of the mediastinum: A rare tumor at a rare site. J Pioneer Med Sci. 20I6;6:100-3.

5. Hazzard C, Kaufman A, Flores R. Mediastinal Tumours. In: International Association for the Study of Lung Cancer (IASLC). Thoracic Oncology. 2nd ed. Philadelphia: Elsevier; 20I8. Fecha de consulta: 8 de enero de 20I9. Disponible en:

https:/www.clinicalkey.es/service/content/pdf/watermarked/3-s2.o-B9780323523578000548.pdf?locale=es_ES

6. Miranda E, Cifuentes LK, Vélez JG, Pinzón VA. Enfoque inicial de las alteraciones mediastinales: Revisión de sus referencias anatómicas radiográficas. Rev. Colomb. Cardiol. 20I8;25:353-4I6.

https://doi.org/Io.IoI6/j.rccar.20I7.Io.oro

7. Dwivedi AN, Goel K, Tripathi S, Garg S, Rai M. Primary neuroendocrine mediastinal tumor presenting with carcinoid syndrome and left supraclavicular lymphadenopathy. Clinic-radiological and pathological features. J Cancer Res Ther. 2013;9:278-80. https://doi.org/IO.4IO3/0973-I482.I40775

8. Oliveira EH, Oliveira CH, Di Pietro D. Fatores associados à sobrevida de pacientes com tumores de mediastino. Arq. Catarin Med. 2017;46:2-I6.

9. Kitami A, Sano F, Ohashi S, Suzuki K, Uematsu S, Suzuki T, et al. The usefulness of positron-emission tomography findings in the management of anterior mediastinal tumours. Ann Thorac Cardiovasc Surg. 2017;23:26-30. https://doi.org/I0.576I/atcs.oa.I6-00205

Io. Rodriguez C, Arce-Aranda C, Amarilla L, Andreo T, Araujo D, Arzamendia L, et al. Características clínicas y patológicas de los tumores de mediastino en un hospital universitario. Cir. Parag. 2013;37:22-5.

II. Ríos-Rodríguez A, Capin-Sarria N, Torres-Aja L. Tumores de mediastino: informe sobre 29 pacientes. Rev Cubana Cir. 2008;47:7p. Fecha de consulta: 23 de enero de 20I9. Disponible en: http://scielo.sld.cu/scielo.php?scrip$\mathrm{t}=$ sci_arttext\&pid=So034-74932008000400oo4\&lng=es
I2. Mederos-Curbelo ON, Barrera-Ortega JC, Villafranca-Hernández O, Gómez-Guirola L, Mederos-Trujillo OL. Morbilidad de las afecciones quirúrgicas del mediastino. Rev Cubana Cir. 20II;50:45I-6I.

I3. Salazar Díaz SP, Calderón Villa F, Moya Paredes E, Poveda Granja S. Tumores mediastínicos gigantes. Estudio de una década, Hospital Carlos Andrade Marín, Quito. Rev CIEZT Clínica y Cirugía. 20I4;I2:I-7.

I4. McCool FD. Enfermedades del diafragma, la pared torácica, la pleura y el mediastino. En: Goldman L, Schafer AI. Goldman-Cecil. Tratado de Medicina Interna. $25^{\mathrm{a}}$ ed. España: Elsevier; 20I7. Fecha de consulta: I7 enero de 2019. Disponible en:

https://www.clinicalkey.es/service/content/pdf/watermarked/3-s2.0-B9788491130338000999.pdf?locale=es_ES

I5. Reimão SM, Colaiacovo R, Camunha MAR, Amancio TT, Segatelli V, Paulo GA. Tumor mediastinal: nem sempre um linfoma. Einstein (São Paulo). 20I7;15:376-377. https://doi.org/Io.I590/sı679-450820I7ai398I

I6. Luketich JD, Ginsberg RJ. The current management of patients with mediastinal tumours. Adv Surg. 20I7;30:3II-32.

17. Jiménez-Fuente E, Chinchilla-Trigos LA, Herrera-Gómez Á, Avilés-Salas A, Martínez-Hernández H. Sarcoma de mediastino. Serie de casos y revisión de la literatura. Departamento de Oncología Torácica. Instituto Nacional de Cancerología. INCan. Rev Fac Med (Méx) [Internet]. 20I7;60:6-I7.

I8. Lee C, Arce-Aranda C, Torrent MA, Leiva A, Flor R, Soskin-Reidman A, et al. Mediastinoscopia cervical estándar para el diagnóstico de enfermedad mediastinal. Cir. Parag. 2018;42:I2-7. https://doi.org/I0.18004/sopaci.20I8.diciembre.I2-I7

19. Fuentes-Valdés E. Mediastinotomía anterior y mediastinoscopia cervical en el diagnóstico de las lesiones tumorales mediastinales. Rev Cubana Cir. 2005;44:[6p.]. Fecha de consulta: 9 de febrero de 20I9. Disponible en: http://scielo.sld.cu/scielo.php?script=sci_arttext\&pi$\mathrm{d}=$ So034-74932005000I00004\&lng=es

20. Chaveco-Bautista D, Babié-Reyes BE, Frómeta-Luna FI, Ronda-León MF, Rodríguez-Griñán A. Linfoma mediastinal en una lactante. MEDISAN. 20I0;I4:709-I3.

2I. De los Reyes I, Saavedra C, Quijano S, Varón A, Moreno CE, Jurado M. Tumores malignos de mediastino en niños: un problema clínico urgente. Biomédica. 20I0;30(Supl):27-3I.

https://doi.org/I0.7705/biomedica.v30io.820

22. Hernández-Benedicto R, Pila-Pérez R, Rosales-Torres P. Tumor de células germinales primario de mediastino (seminoma de mediastino). Rev. Arch Med Camagüey. 2019;23:95-103.

23. Chahoud J, Zhang M, Shah A, Lin SH, Pisters LL, TuS $\mathrm{M}$. Managing seminomatous and nonseminomatous 
germ cell tumors. Curr Opin Oncol. 20I8;30:I8I-8. https://doi.org/I0.I097/CCO.0000000000000446

24. Weissferdt A, Rodríguez-Canales J, Liu H, Fujimoto J, Wistuba II, Moran CA. Primary mediastinal seminomas: a comprehensive immunohistochemical study with a focus on novel markers. Human Pathology. 2015;46:376-83.

https://doi.org/IO.IoI6/j.humpath.20I4.II.009

25. Pham RL, Ray A, Margraf LR. Mixed nonseminomatous germ cell tumor with rhabdomyosarcomatous malignant transformation in a pediatric patient. Indian J Med Paediatr Oncol. 20I8;39:250-3. https://doi.org/IO.4IO3/ijmpo.ijmpo_207_I7

26. Xu J, Zhao J, Geng S, Wang Q, Wang P, Zhang C, et al. Primary seminoma arising in the middle mediastinum: A case report. Oncol Letters. 20I6;12:348-50. https://doi.org/IO.3892/ol.20I6.4575

27. Charlotte J, Mathilde D, Emmanuel D, Lionnel G. Bulletin du Tumeurs germinales primitives du médiastin: expérience de l'Institut de cancérologie de Lorraine sur une période de 20 ans (1990-20I2). Bulletin du Cancer [Internet]. 20I4;IOI:[7p.]. Fecha de consulta: I2 de enero de 20I9. Disponible en:

https://www.sciencedirect.com/science/article/abs/pii/ Sooo7455II5303039

28. Fuentes-Valdés E, Corona-Mancebo SB. Tumores primarios malignos de tráquea y bronquios principales. Rev Cubana Cir. 2002;4I:I76-84.

29. Cúneo L, Dutruel SP, Cellerino AV, Salazar-Cottone $\mathrm{SN}$, Badano F, Maio Gl, et al. Tumor germinal no seminomatoso del mediastino con invasión pulmonar. Rev Argent Radiol. 2008;72:87-92.

30. García-Castañeda H, Borrazas-González MC, Fernández-Fernández M. Teratoma mediastinal anterior. Rev Cub Med Mil. 2016;45:229-34.

3I. Martínez-Navarro J, Nápoles-Torres Y, Villafuerte D. Teratocarcinoma mediastinal. Presentación de un caso. Revista Electrónica de la Autopsia. 20I7;I4:I7-20.

32. Elstner K, Granger E, Wilson S, Kumaradevan N, Chew $\mathrm{M}$, Harris C. Schwannoma of the pulmonary artery. Heart, Lung and Circulation. 2013;22:23I-3. https://doi.org/IO.IOI6/j.hlc.20I2.07.0I2

33. Weng CH, Chen JB, Wang J, Wu CC, Yu Y, Lin TH. Case report. Surgically curable non-iron deficiency microcytic anemia: Castleman's disease. Onkologie. 20II;34:456-8. https://doi.org/IO.II59/00033I283
34. Vega-Lorenzo Y, Hidalgo-Ávila M, Martínez-Lorenzo F, García-Martín D, Aparicio-Álvarez FE, González-Díaz EC. Síndrome mediastinal. Presentación de un caso. MEDICIEGO. 20I6;22:6I-7.

35. Mederos-Curbelo ON, Menchaca-Díaz JL, Barreras-Ortega JC, Cantero-Ronquillo A, Valdés-Jiménez J. Quiste pericárdico gigante en el adulto. Reporte de I caso. Rev Cubana Cir. 200I;40:312-4.

36. Nakhla SG, Sundararajan S. A rare case of primary anterior mediastinal yolk sac tumor in an elderly adult male. Case Rep Oncol Med. 2016; 20I6: 896I486.

https://doi.org/IO.II55/20I6/896I486

37. Putnam JB. Pulmón, pared torácica, pleura y mediastino. En: Townsend CM, Beauchamp RD, Evers BM, Mattox KL. Sabiston. Tratado de Cirugía. 20 $0^{\mathrm{a}}$ ed. España: Elsevier. Fecha de consulta: I7 de Junio de 2018. Disponible en:

https://www.clinicalkey.es/service/content/pdf/watermarked/3-s2.o-B978849II31328000573.pdf?locale=es_ES

38. Cao J, Zhou Y, Zou F, Ma J-A, Hu Ch. Intensity modulated radiation therapy to treat primary female mediastinal seminoma and massive pericardial effusion: A case report. Oncol Lett. 2017;13:1299-I302. https://doi.org/I0.3892/ol.2017.5555

39. Yutaro T, Takehiko O, Takashi N, Daichi K, Takahiro $\mathrm{K}$, Hidetoshi A, et al. A study of patients with primary mediastinal germ cell tumours treated using multimodal therapy. Adv Urol. 2017;2017:14046I0. https://doi.org/IO.II55/20I7/I4046IO

40. Amadeo F, Alessandra B, Augusto O. Mediastinal germ cell tumours: new therapeutic insights. J Thorac Dis. 20I7;9:3620-22. https://doi.org/I0.2I037/jtd.20I7.09.46

4I. Lucia N, Matti A, Gillian V, Landan F, Martín G, Kim N Ch. Molecular dissection of primary mediastinal germ cell tumours. J Clin Oncol. 2017;35(suppl):4I7. https://doi.org/IO.I200/JCO.2017.35.6_suppl.4I7

42. Keating J, Judy R, Newton A, Singhal S. Near-infrared operating lamp for intraoperative molecular imaging of a mediastinal tumor. BMC Med Imaging. 2016;16:15. https://doi.org/IO.II86/sI2880-0I6-OI2O-5

43. Furuto $Y$, Hashimoto $H$, Horiuti $H$, Shibuya $Y$. Membranoproliferative glomerulonephritis-like findings for TAFRO syndrome, associated with an anterior mediastinal tumor. Medicine. 2018;97:eIIO57. https://doi.org/I0.I097/MD.ooooooooooolio57 